\title{
Synthesis and Antiviral Activity of 2'( $\beta$ )-Hydroxymethylated Carbodine Analogues Against Hepatitis C Virus
}

\author{
Joon Hee Hong and Chang-Hyun $\mathrm{Oh}^{\dagger}$,
}

\author{
BK-21 Project Team, College of Pharmacy, Chosu Chiversity, Kwangin 501-759. Korea \\ -Biomaterials Research Center, Korea Institute of Science and Technology, Seoul 130-650, Korea. "E-mail: chohaikist. re.kr \\ Received August 1t. 2009. Accepted September 15, 2009
}

\begin{abstract}
$2^{\prime}(\beta)$-Hydroxymethylated adenosine is a potent and selective inhibitor of hepatitis C virus (HCV) replication. It targets the RNA-dependent RNA polymerase of HCV, NS5B. Synthesis and antiviral evaluation of carbocyclic versions are described. The cyclopentene intermediate (9) was successfully synthesized through sequential Johnson-Claisen orthoester rearrangement and ring-closing metathesis (RCM). Coupling of bases via a Pd( 0 ) catalyst, selective dihydroxylation, and desily lation y ielded the target nucleoside analogues. The compounds 17 and 18 were assayed for their ability to inlubit $\mathrm{HC} V \mathrm{RNA}$ replication in a subgenomic replicon $\mathrm{Hul} 7 \mathrm{cell}$ line and showed moderate antiviral activity with toxicity up to 20.0 and $24.7 \mathrm{\mu g} / \mathrm{mL}$, respectively.
\end{abstract}

Key Words: Carbodine. Anti-HCV agent. Substituted carbocyclic nucleoside

\section{Introduction}

Hepatitis $\mathrm{C}$ virus $(\mathrm{HCV})^{1.3}$ is a leading cause of chronic hepatitis. liver cirrhosis and hepatocellular carcinoma. Current therapy based on pegylated interferon and ribavirin is often poorly tolerated and effective in only $50 \%$ of patients. More effective therapeutic agents against $\mathrm{HCV}$ are needed.

Nucleoside analogues are the drugs of choice in the treatment of viral infection. and were syththesized and evaluated for anti-HCV activity: ${ }^{4-6}$ These nucleosides are incorporated into proviral RNA and act as chain terminators. Modification around the 2'-hydroxy group of the ribose in natural ribonucleosides can produce effective RNA chain temination. ${ }^{8}$ For example. $2^{\prime}-C$-methy lcỵtidine $(\mathbf{1})^{9}$ and $2^{\prime}-C-$ methyladenosine $(2)^{10}$ are potent anti-HCV agents in clinical trials.

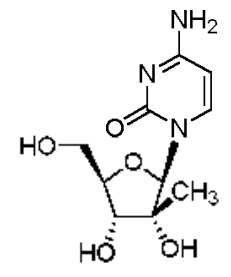

2'-C-methylcytidine

1

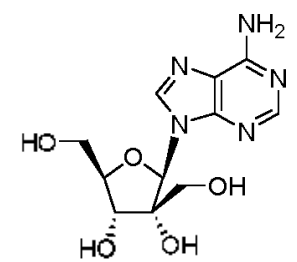

2'-C-hydroxymethyladenosine

3

Figure 1. Structures of potent anti-HCV agents and $2^{\prime}(\beta)$-carbodine analogues (17 and 18 ).

2'-C-methyladenosine

2

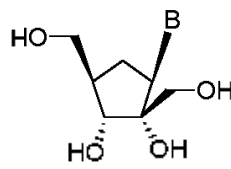

17: $B=$ cytosine

18: $B=$ adenine
In 2006, Yoo et al. reported the synthetic procedure and potent anti-HCV activity of $2^{\prime}-C^{-}$-hy'droxy'methyladenosine (3). ${ }^{1]}$ Based on these findings, we designed and synthesized novel carbocyclic classes of mucleosides comprising 2'-C-hydroxymethylated carbodine analogues (Fig. 1).

\section{Results and Discussion}

Chemistry. The $\gamma . \hat{\delta}$-unsaturated ethyl ester 5 was readily synthesized from the commercially available 2-butene-1,4-diol (4) using the reported procedure." The ester 5 was reduced to the corresponding aldehyde 6 using DIBAL-H in toluene at $-78^{\circ} \mathrm{C}$. The condensation of 6 with the lithium reagent prepared from 3 equivalents of 2-bromo-ally loxy-tert-butyldimethylsilane 7 and 2.5 equivalents of butyllithium in THF at $-110^{\circ} \mathrm{C}$ yielded diene analogue $8^{1 / 3}$ (Scheme 1 ).

The diene analogue $\mathbf{8}$ diastereomeric mixture was subjected to ring-closing metathesis (RCM) conditions ${ }^{1+18}$ to yield cyclo-

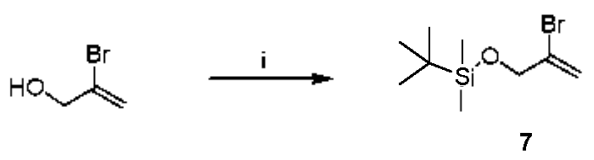

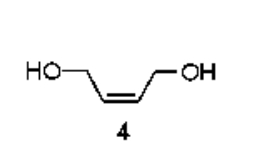

1,4-Dihydroxy-2-butene
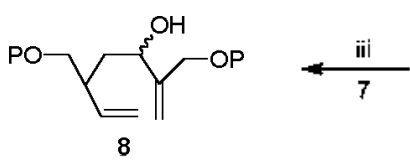<smiles>CC#CCCCCC(=O)OCc1ccccc1</smiles>

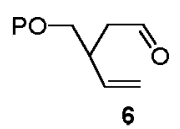

Scheme 1. Reagents and conditions: i) TBDMSCI, imidazole, $\mathrm{CH}_{2}-$ $\mathrm{Cl}_{2}, 0^{\circ} \mathrm{C}$ : ii) DIBAL-H, toluene, $-78^{\circ} \mathrm{C}$; iii) 7,2 -bromo-allyloxy-tertbutylmethysilane, n-butyllithium, $-110^{\circ} \mathrm{C}$, THF. 


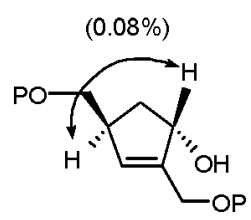

$9(\alpha)$

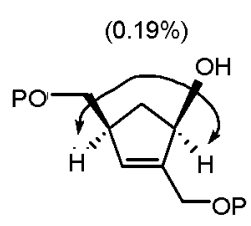

$9(\beta)$
Figure 2. NOE comparison of compounds $9(\alpha)$ and $9(\beta)$.

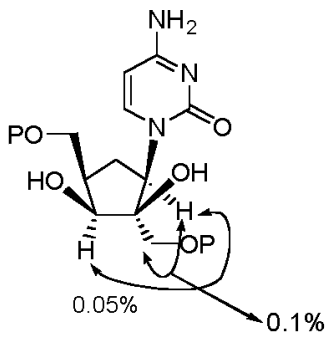

15

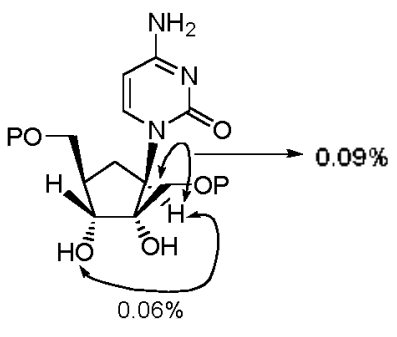

13
Figure 3. NOE comparison of compound 13 and $\mathbf{1 5}$

pentenol derivatives $9 \alpha$ and $9 \beta$. The stereochemical differences between the two isomers were determined by NOE comparisons. Irradiation of $C_{1}-\mathrm{H}$, produced different NOE patterns at the proximal hydrogens of compound $9 \beta$ [methw/oxy-H $(0.19 \%)]$ compared to compound $9 \alpha$ [methyloxr-H $(0.08 \%)$ ] (Fig. 2).

Compound $9 \beta$ was converted into 10 using ethyl chloroformate. and readily coupled with cytosine and adenine by ally lic functionalization using a palladium cataly st adduct to generate nucleoside analogues $\mathbf{1 1}$ and 12. respectively. Carbodine analogues were obtained by oxidation of the protected nucleosides 11 and 12 to yield dihy'droxylated 13 and $\mathbf{1 4}$ as major reaction products with a small amount of $\mathbf{1 5}$ and $\mathbf{1 6}$. respectively: Stereochemical differences were determined by NOE experiments. Irradiation of $C_{1}-\mathrm{H}$ of compound 15 produced different NOE patterns at the proximal hydrogens such as 2'methyloxy-H $(0.10 \%)$ and $3^{\prime}-\mathrm{H}(0.05 \%)$. compared with com-

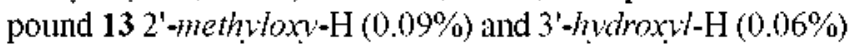
(Fig. 3). Bulky groups such as silylated hydroxymethyl group and nucleosidic bases (adenine and cytosine) reinforce the steric lindrance of the $\beta$-faces.$^{19.2(1)}$ Removal of the protecting silyl group of 13 and 14 was performed by treatment with tetrabultylammonium fluoride (TBAF) to yield target nucleosides 17 and 18 respectively (Scheme 2 ).

Antiviral Activity. The newly synthesized nucleoside analogues were assayed for anti-HCV activity using an in vitro assay system that is suitable for monitoring anti-HCV activities of compounds. This system is composed of a human hepatocarcinoma cell line (Huh-7) supporting multiplication of a HCV replication, and the results are summarized in Table l. These cells contain a HCV subgenomic replicon RNA encoding a luciferase reporter gene as a marker. The antiviral potency of the mucleoside analogies against the HCV replicon is expressed as $\mathrm{EC}_{5 \mathrm{j})}$, which was quantified by a luciferase assay after a two-days incubation period with the tested compound. To confirm the anti-HCV potency of compounds. subgenomic
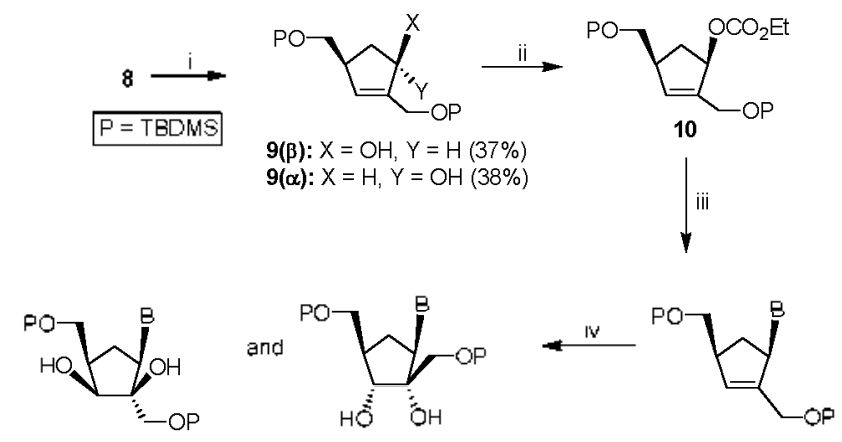

15: $B=$ cytosine 16: $\mathrm{B}=$ aderine

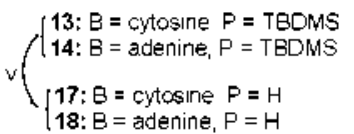

11: $B=$ cytosine 12: $\mathrm{B}=$ adenıne

18: $\mathrm{B}=$ adenine, $\mathrm{P}=\mathrm{H}$

Scheme 2. Reagents and conditions: i) Grubb's catalyst (II), benzene: ii) $\mathrm{ClCO}_{2} \mathrm{Et}$, pyridine DMAP, iii) cy tosine, adenine, $\mathrm{Pd}_{2}(\mathrm{dba})_{3} \mathrm{CH}-$ $\mathrm{CL}_{3}, \mathrm{P}(\mathrm{O}-\mathrm{i}-\mathrm{Pr})_{3}, \mathrm{NaH}, \mathrm{THF} / \mathrm{DMSO}$ iv) $\mathrm{OsO}_{4}, \mathrm{NMO}$; $)$ TBAF, THF/ $\mathrm{CH}_{3} \mathrm{CN}, \mathrm{rt}$.

Table 1. Anti-HCV activity of the newly synthesized compounds 17 and 18 .

\begin{tabular}{ccc}
\hline Compound No. & $\begin{array}{c}\text { Anti-HCV } \\
\mathrm{EC}_{50}(\mu \mathrm{g} / \mathrm{mL})\end{array}$ & $\begin{array}{c}\text { Cytotoxicity } \\
\mathrm{CC}_{50}(\mu \mathrm{g} / \mathrm{mL})\end{array}$ \\
\hline $\mathbf{1 7}$ & 11.7 & 20.0 \\
$\mathbf{1 8}$ & 18.2 & 24.7 \\
$2^{\prime}-\mathrm{C}-\mathrm{Me}-\mathrm{C}$ & 3.7 & $>50$ \\
\hline
\end{tabular}

$2^{\prime}-C-M e-C: 2^{\prime}-C$-Methylcrtidine. $\mathrm{EC}_{5 n}(\mu \mathrm{g} \mathrm{mL})$ : concentration required to inhibit $50^{\circ} \mathrm{o}$ of the sirus induced cytopathicity. $\mathrm{CC}_{5 u}(\mu \mathrm{g} \mathrm{mL})$ : concentration required to reduce cell riability by $50^{\circ}$.

replicon RNA levels were quantified by real-time RT-PCR analysis. In addition, the associated cytotoxicity (expressed as $\mathrm{CC}_{5(1}$ in Table 1) was evaluated in a tetrazolium (XTT)-based assay. $2^{\prime}-C$-Methylcytidine (1) was selected as the reference standard due to its stnucture similarity to the newly synthesized compounds. Both compounds 17 and 18 inhibited the replication of the replicon in Hull-7 cells by $50 \%$ at concentrations of $11.7 \mu \mathrm{g} / \mathrm{mL}$ and $18.2 \mu \mathrm{g} / \mathrm{mL}$. respectively:

In sunmary, on the basis of potent anti-HCV activity of 2'C-hydroxymethy ladenosine. we have accomplished the stereoselective synthesis of its carbodine derivatives starting from 2-butene-1.4-diol. Compounds 17 and 18 exhibited good anti$\mathrm{HCV}$ activity. indicating that the hydroxymethyl group at 2'position of carbodine system makes the conformation to be favorable for interaction with $\mathrm{HCV}$ poly merase.

\section{Experimental Section}

Melting points (mp) were measured on a Mel-temp II laboratory device and are uncorrected. NMR spectra were recorded using a JEOL 300 Fourier transform spectrometer (JEOL. Tokyo. Japan). UV spectra were obtained using a Beckman DU-7 spectrophotometer (Beckman. South Pasadena. CA. USA). The elemental microanalyses were performed using a Perkin-Elmer 2400 analyzer (Perkin-Elmer, Nonwalk. CT, USA).

( \pm )-3-(tert-Butyldimethylsilanyloxymethyl)-pent-4-enal 
(6). To a solution of $5(2.5 \mathrm{~g} .9 .17 \mathrm{mmol})$ in toluene $(60 \mathrm{~mL})$. DIBAL-H $(6.73 \mathrm{~mL}, 1.5 \mathrm{M}$ solution in toluene. $10.10 \mathrm{mmol})$ was added slowly at $-78^{\circ} \mathrm{C}$. and stirred for $15 \mathrm{~min}$ at the same temperature. Methanol ( $7 \mathrm{~mL}$ ) was added and the reaction mixture was stirred at room temperature for $2 \mathrm{~h}$. The resulting solid was filtered through celite. The filtrate was evaporated under reduced pressure. and the residue was purified by column chromatography (silica gel. ethyl acetate-hexanes $1: 30 \mathrm{v} / \mathrm{v}$ ) to yield compound $6(1 .+4 \mathrm{~g}, 69 \%)$ as a colorless oil: ${ }^{\mathrm{l}} \mathrm{H}$ NMR $\left(\mathrm{CDCl}_{3 .} 300 \mathrm{MHz}\right.$ ): $\delta 9.72$ (s. $\left.1 \mathrm{H}\right), 5.76-5.65$ (m. $\left.1 \mathrm{H}\right), 5.11-$ $5.05(\mathrm{~m} .2 \mathrm{H}), 3.65(\mathrm{dd}, J=9.8$ and $4.8 \mathrm{~Hz} .1 \mathrm{H}), 3.43(\mathrm{dd} . J=$ 9.8 and $2.6 \mathrm{~Hz}, \mathrm{lH}) .2 .76(\mathrm{~m}, \mathrm{lH}), 2.62-2.5 \mathrm{l}(\mathrm{m}, \mathrm{lH}) .2 .43-$ $2.32(\mathrm{~m}, \mathrm{lH}), 0.81$ (s. 9H). 0.01 (s. $6 \mathrm{H}){ }^{13} \mathrm{C} \mathrm{NMR}\left(\mathrm{CDCl}_{3,75}\right.$ $\mathrm{MHz}): \delta 202.1 .137 .7 .116 .4,65.8,45.5 .41 .1,25.8,18.3 .-5.5$.

(2-Bromoallyloxy)-tert-butyldimethylsilane (7). tert-Butylchlorodimethylsilane $(6.05 \mathrm{~g} .40 .15 \mathrm{mmol})$ was added to a stirred solution of 2-bromoallyl alcohol $(5.0 \mathrm{~g}$. $36.5 \mathrm{mmol})$ and imidazole $(3.85 \mathrm{~g} .56 .57 \mathrm{mmol})$ in $\mathrm{CH}_{2} \mathrm{Cl}_{2}(150 \mathrm{~mL})$ at 0 ${ }^{\circ} \mathrm{C}$. The reaction mixture was stirred at room temperature for $4 \mathrm{~h}$. and then evaporated under reduced pressure. The residue was partitioned between water and ethyl acetate and the organic layer was dried over $\mathrm{MgSO}_{+}$and filtered. After evaporation of the organic solvent, the residue was purified by column chromatography (silica gel. ethyl acetate-hexanes $1: 40 \mathrm{v} / \mathrm{v}$ ) to yield compound $7(8.53 \mathrm{~g} .93 \%)$ as a colorless sy rup: ${ }^{\mathrm{l}} \mathrm{H} \mathrm{NMR}$ $\left(\mathrm{CDCl}_{3} .300 \mathrm{MHz}\right): \grave{o} 5.6 \mathrm{l}-5.55(\mathrm{~m} .2 \mathrm{H}) .4 .61$ (d. $J=0.6 \mathrm{~Hz}$. $2 \mathrm{H}) .0 .82$ (s. 9H). 0.12 (s.6H).

rel-(3R and $3 S, 5 R)-2,5$-Bis-(tert-Butyldimethylsilanyloxymethyl)hepta-1,6-dien-3-ol (8). To a solution of compound 7 $(4+1 \mathrm{mg}, 1.755 \mathrm{mmol})$ in dry THF $(5 \mathrm{~mL})$ cooled at $-110^{\circ} \mathrm{C}$ (ether and liquid nitrogen). butỵllithium $(0.91 \mathrm{~mL}$. $1.6 \mathrm{M}$ solution in hexanes. $1.46 \mathrm{mmol}$ ) was slowly added over 5 min under an argon atmosphere. After stirring for $15 \mathrm{~min}$ at the same temperature a solution of 6 (133 $\mathrm{mg} .0 .585 \mathrm{mmol})$ in dry THF $(0.5 \mathrm{~mL})$ was slowly added to the reaction mixture over $5 \mathrm{~min}$. and stirred for $15 \mathrm{~min}$ at the same temperature. The reaction mixture was quenched with saturated aqueous $\mathrm{NH}_{4} \mathrm{Cl}$ solution $(2 \mathrm{~mL}$ ) and warmed slowly to room temperature. The mixture was extracted twice with diethyl ether ( $5 \mathrm{~mL}$ each) and the combined organic layer extracts were washed with brine. dried over $\mathrm{MgSO}_{4}$. filtered and evaporated under reduced pressure. The residue was purified by column chromatography (silica gel. ethyl acetate-hexanes $1: 30 \mathrm{v} / \mathrm{v}$ ) to yield a diastereomeric misture of 8 ( $157 \mathrm{mg} .67 \%$ ) as a colorless oil: ${ }^{1} \mathrm{H} \mathrm{NMR}\left(\mathrm{CDCl}_{3}\right.$. $300 \mathrm{MHz})$ : ò $5.71(\mathrm{~m}, \mathrm{HH}) .5 .25-5.14(\mathrm{~m} .4 \mathrm{H}) .3 .92(\mathrm{~m}, \mathrm{lH})$. $3.73-3.67(\mathrm{~m} .2 \mathrm{H}) .3 .5 \mathrm{l}(\mathrm{m} .2 \mathrm{H}) .2 .27(\mathrm{~m} . \mathrm{lH}) .1 .52-1.47(\mathrm{~m}$. $2 \mathrm{H}) .0 .82(\mathrm{~m} .18 \mathrm{H}), 0.01$ (s. $12 \mathrm{H}) .{ }^{13} \mathrm{C} \mathrm{NMR}\left(\mathrm{CDCl}_{3} .75 \mathrm{MHz}\right)$ : ò $154.5 .143 .6,112.7,107.4 .71 .8,70.2 .67 .2,39.8,36.3,25.6$. 18.6. -5.4 .

ret-(1R,+S)-2,4-Bis-(tert-butyldimethylsilanyloxymethyl) cyclopent-2-enol (9ß) and rel-(1S,4S)-2,4-bis-(tert-lutyldimethylsilanyloxymethyl)cyclopent-2-enol (9a). To a solution of $8(2.5 \mathrm{~g} .6 .2+\mathrm{mmol})$ in dry benzene $(12 \mathrm{~mL})$. second generation Grubbs' catalyst (40 mg. $0.0471 \mathrm{mmol}$ ) was added. The reaction mixture was refluxed overnight and then evaporated under reduced pressure. The residue was purified by column chromatography (silica gel. ethyl acetate-hexanes 1:25 v/v) to yield $9 \beta(860 \mathrm{mg} .37 \%$ ) and $9 \alpha(883 \mathrm{mg} .38 \%$ ). Compound $9 \beta$ :
${ }^{\mathrm{J}} \mathrm{H} \mathrm{NMR}\left(\mathrm{CDCl}_{3} .300 \mathrm{MHz}\right): \delta 5.54(\mathrm{~d} . J=5.8 \mathrm{~Hz} .1 \mathrm{H}), 4.45$ (s. $2 \mathrm{H}$ ). 4.09 (dd. $J=5.4$ and $2.4 \mathrm{~Hz} . \mathrm{IH}$ ). 3.72 (dd. $J=10.2$ and $6.8 \mathrm{~Hz}, \mathrm{IH}) .3 .59$ (dd, $J=10.2$ and $8.2 \mathrm{~Hz}, \mathrm{lH}$ ). $2.48-2.40$ (dd, $J=10.0$ and $8.2 \mathrm{~Hz}, 1 \mathrm{H}$ ), $2.02-1.95$ (dd. $J=10.0$ and 6.8 Hz. IH). 0.82 (s. 18H). 0.02 (s. 12H). ${ }^{13} \mathrm{C} \mathrm{NMR}$ (CDCl 35 $\mathrm{MHz})$ : ô 1+3.5. 126.2, 72.9, 69.9, 67.5. 41.1. 30.7. 25.7. 18.3, -5.7. Analysis for $\mathrm{C}_{19} \mathrm{H}_{40} \mathrm{O}_{3} \mathrm{Si}_{2}(514.91)$. Calcd: $\mathrm{C} .61 .23: \mathrm{H}$. 10.82: Found: C. 61.34: H. 10.77. Compound 9ק: ${ }^{1} \mathrm{H}$ NMR $\left(\mathrm{CDCl}_{3} .300 \mathrm{MHz}\right): \hat{o} 5.57(\mathrm{~d} . J=6.2 \mathrm{~Hz}, 1 \mathrm{H}) .+48(\mathrm{~d}, J=2.1$ Hz. $2 \mathrm{H}$ ) $4.0 \mathrm{l}(\mathrm{d}, J=5.0 \mathrm{~Hz}, \mathrm{lH}) .3 .75$ (dd $J=9.8$ and $6.2 \mathrm{~Hz}$. $1 \mathrm{H}) .3 .61$ (dd. $J=9.8$ and $8.0 \mathrm{~Hz}, 1 \mathrm{H}), 2.52-2.45(\mathrm{dd}, J=10.2$ and $7.8 \mathrm{~Hz}, \mathrm{IH}) .2 .05-1.99$ (dd. $J=10.0$ and $8.2 \mathrm{~Hz}, \mathrm{lH}) .0 .8 \mathrm{l}$ (m, 18H), 0.01 (s. $12 \mathrm{H}) .{ }^{13} \mathrm{C} \mathrm{NMR}\left(\mathrm{CDCl}_{3} .75 \mathrm{MHz}\right)$ : ô $1+3.7$. 126.5, 71.8, 70.2, 68.2, 42.0. 31.4, 25.5, 18.7, -5.4. Analy'sis for $\mathrm{C}_{19} \mathrm{H}_{40} \mathrm{O}_{2} \mathrm{Si}_{2}(519.41)$. Calcd.: C. 61.23: H. 10.82: Found: C, 61.17 ; H. 10.90

rel-(1R,4S)-1-Ethoxycarbonyloxy-2,4-bis-(tert-butyldimethylsilyloxymethyl)cyclopent-2-ene (10). To a solution of compound $9 \beta$ ( $1.77 \mathrm{~g} .4 .68 \mathrm{mmol}$ ) in anhydrous py ridine (15 $\mathrm{mL}$ ). ethyl chloroformate $(547 \mathrm{mg} .5 .04 \mathrm{~nm}$ ol) and DMAP (49 $\mathrm{mg}, 0.4$ nmol) were added. The reaction mixture was stirred overnight at $60^{\circ} \mathrm{C}$. The reaction mixture was then quenched using a saturated $\mathrm{NaHCO}_{3}$ solution $(0.5 \mathrm{~mL})$ and evaporated under reduced pressure. The residue was partitioned between water and ethyl acetate and the organic layer was separated. The aqueous layer was extracted with ethyl acetate. and the combined organic layer extracts were washed with brine, dried over $\mathrm{MgSO}_{4}$ and filtered. The organic solvent was evaporated under reduced pressure and the residue was purified by column chromatography (silica gel, ethyl acetate-hexanes $1: 25 \mathrm{v} / \mathrm{v}$ ) to yield compound $10(1.66 \mathrm{~g} .80 \%)$ as a colorless synup. ${ }^{\mathrm{l}} \mathrm{H}$ NMR $\left(\mathrm{CDCl}_{3}, 300 \mathrm{MHz}\right): \delta 5.57$ (d. $\left.J=6.2 \mathrm{~Hz}, 1 \mathrm{H}\right), 4.85$ (dd, $J=6.8$ and $4.2 \mathrm{~Hz}, 1 \mathrm{H}) .4 .48(\mathrm{~d}, J=1.8 \mathrm{~Hz}, 2 \mathrm{H}) .+.2 \mathrm{l}(\mathrm{t} . J=7.2 \mathrm{~Hz} .2 \mathrm{H})$. 3.69 (dd $J=9.8$ and $6.4 \mathrm{~Hz}, \mathrm{lH}$ ). 3.46 (dd $J=9.8$ and $7.8 \mathrm{~Hz}$. lH). $2.78-2.69(\mathrm{dd}, J=10.0$ and $6.8 \mathrm{~Hz}, 1 \mathrm{H}), 2.02-1.94(\mathrm{dd} . J$ $=10.0$ and $7.4 \mathrm{~Hz}, \mathrm{IH}), 1.29(\mathrm{t} . J=7.2 \mathrm{~Hz}, 3 \mathrm{H}), 0.8 \mathrm{l}(\mathrm{m}, \mathrm{l} \mathrm{H})$. $0.01(\mathrm{n}, 12 \mathrm{H}){ }^{13} \mathrm{C} \mathrm{NMR}\left(\mathrm{CDCl}_{3}, 75 \mathrm{MHz}\right):$ ô $155.1,144.5$. $127.8,79.9 .71 .6 .67 .7,64.3,41.2 .32 .6 .25 .7,18.6,13.9 .-5.6$. Analysis for $\mathrm{C}_{22} \mathrm{H}_{44} \mathrm{O}_{5} \mathrm{Si}_{2}(444.75)$. Calcd: C. 59.41: $\mathrm{H}, 9.97$ : Found: C. $59.55: \mathrm{H}, 10.02$.

rel-(1'R,+'S)-1-[2,4-Bis-(tert-butyldimethylsilyloxymethyl) cyclopent-2-en-1-yl]cytosine (11). Cytosine (104 mg. 0.936 mmol) was added to a solution of hexane-washed $\mathrm{NaH}(22.4$ $\mathrm{mg}, 0.936 \mathrm{mmol})$ in anlydrous DMSO $(5.0 \mathrm{~mL})$. The reaction nisture was stirred for $30 \mathrm{~min}$ at $50-55^{\circ} \mathrm{C}$ and cooled to roon temperature. Simultaneously. $\mathrm{P}(\mathrm{O}-\mathrm{i}-\mathrm{Pr})_{3}(78 \mathrm{mg} .0 .37+\mathrm{mmol})$ was added to a solution of $\mathrm{Pd}_{2}(\mathrm{dba})_{3} \cdot \mathrm{CHCl}_{5}(50 \mathrm{mg} .4 .8 \mu \mathrm{mol})$ in anlydrous THF (4.5 mL), which was stirred for $30 \mathrm{~min}$. The catalyst solution in THF and 10 (373.6 $\mathrm{mg} .0 .84 \mathrm{mmol})$ dissolved in anhydrous THF $(4.0 \mathrm{~mL})$ was sequentially added to the cytosine solution in DMSO. The reaction mixture was refluxed ovemight, and then cooled and quenched with water $(2.0 \mathrm{~mL})$. The solvent was evaporated under reduced pressure and the residue was purified by column chromatography (silica gel. MeOH/Hexane/EtOAc, $0.1: 3: 1$ ) to yield compound $11(1+5 \mathrm{mig} .37 \%)$ as a white solid. ${ }^{1} \mathrm{H}$ NMR $\left(\mathrm{CDCl}_{3}, 300\right.$ $\mathrm{MHz}$ ): $0 \hat{0} 7.24$ (d. $J=6.8 \mathrm{~Hz} .1 \mathrm{H}) .5 .78(\mathrm{~d} . J=6.8 \mathrm{~Hz}, 1 \mathrm{H}), 4.57$ (dd $J=5.6$ and $1.2 \mathrm{~Hz}, \mathrm{lH}$ ). 4.42 (s. $2 \mathrm{H}$ ). 3.53 (dd. $J=12.8$ 
and $6.4 \mathrm{~Hz}, 2 \mathrm{H}), 2.40(\mathrm{dd} . J=12.6$ and $8.2 \mathrm{~Hz}, \mathrm{lH}), 2.32(\mathrm{m.} \mathrm{lH})$, 2.01 (dd. $J=12.6$ and $6.4 \mathrm{~Hz} \mathrm{IH}) .0 .82(\mathrm{~m}, \mathrm{l} 8 \mathrm{H}) .0 .0 \mathrm{l}(\mathrm{m} .12 \mathrm{H})$. ${ }^{13} \mathrm{C} \mathrm{NMR}\left(\mathrm{CDCl}_{3 .} .75 \mathrm{MHz}\right)$ : o 165.7 .156 .8 .145 .4 .143 .4 .126 .7$. $91.8,70.7 .67 .4,53.5 .42 .3,30.2 .25 .6,18.4,-5.7$. Analy sis for $\mathrm{C}_{33} \mathrm{H}_{43} \mathrm{~N}_{3} \mathrm{O}_{3} \mathrm{Si}_{2}$ (465.78), Calcd: C. 59.31: H. 9.31: N, 9.02: Found: C, 59.24; H. 9.28; N, 8.95.

rel-(1'R,+'S)-9-[2,4-Bis-(tert-butyldimethylsilyloxymethy]) cyclopent-2-en-1-yl]adenine (12). Adenine nucleoside analogue 12 was synthesized from 10 by a similar procedure as described for 11 . Compound 12 y yield $32 \%:{ }^{1} \mathrm{H} \mathrm{NMR}\left(\mathrm{CDCl}_{3}\right.$. $300 \mathrm{MHz}$ ): $\delta 8.24$ (s. IH). 8.12 (s. $\mathrm{HH}) .5 .69$ (d. $J=6.0 \mathrm{~Hz} . \mathrm{IH})$. $4.6+$ (s. $2 \mathrm{H}), 4.51$ (d. $J=5.8 \mathrm{~Hz} . \mathrm{IH}) .3 .55$ (dd. $J=9.8$ and 4.8 Hz. $\mathrm{lH}$ ). 3.42 (dd. $J=9.8$ and $6.8 \mathrm{~Hz}, \mathrm{IH}) .2 .48$ (dd. $J=12.4$ and $8.0 \mathrm{~Hz}, \mathrm{IH}) .2 .34(\mathrm{~m} . \mathrm{IH}) .2 .03(\mathrm{dd} J=12.4$ and $6.8 \mathrm{~Hz}$. IH) $0.82(\mathrm{~s} .18 \mathrm{H}), 0.01$ (s. $12 \mathrm{H}) .{ }^{19} \mathrm{C} \mathrm{NMR}\left(\mathrm{CDCl}_{3} .75 \mathrm{MHz}\right)$ : ò $156.6,152.4,150.9,145.5,143.6,127.6,117.9,70.7,68.3$. $58.1 .41 .8,29.7 .25 .6,18.4,-5.5$. Analy'sis for $\mathrm{C}_{24} \mathrm{H}_{43} \mathrm{~N}_{5} \mathrm{O}_{2} \mathrm{Si}_{2}$ (489.80). Calcd: C. 58.85: H. 8.85: N. 14.30: Found: C. 58.74: H. 8.92: N, 14.26.

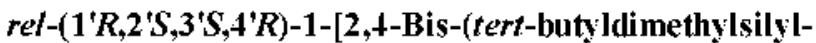
oxymethyl)-2,3-dihydoxycyclo-jentan-1-yl]cy tosine (13) and rel-(1'R,2'R,3'R,+'R)-1-[2,4-bis-(tert-butyldimethyl silyloxymethyl)-2,3-dihydroxy-cyclopentan-1-yl]cytosine (15). To a stirred solution of 12 ( $235 \mathrm{mg} .0 .504 \mathrm{mmol}$ ) in cosolvent ( 4.0 $\mathrm{mL}$. acetone $/$ water $=5: 1 \mathrm{v} / \mathrm{v})$. NMO $(236 \mathrm{mg} .1 .01 \mathrm{mmol})$ and $\mathrm{OsO}_{4}(0.42 \mathrm{~mL}, 4 \%$ aqueous solution $)$ were added. The reaction mixture was stirred overnight at $50^{\circ} \mathrm{C}$. cooled and then quenched with saturated $\mathrm{Na}_{2} \mathrm{SO}_{3}$ solution $(2 \mathrm{~mL}$ ). The resulting solid was removed by filtration through celite and the filtrate was evaporated under reduced pressure. The residue was purified by column chromatography (silica gel. $\mathrm{MeOH} / \mathrm{CH}_{2} \mathrm{Cl}_{2} 1: 7 \mathrm{v} / \mathrm{v}$ ) to yield 13 (143 $\mathrm{mg} .57 \%$ ) and 15 (37 $\mathrm{mg} .15 \%$ ). Compound 13 : ${ }^{1} \mathrm{H}$ NMR (DMSO- $d_{6 .} 300 \mathrm{MHz}$ ): $\delta 7.43$ (d. $J=7.2 \mathrm{~Hz} . \mathrm{IH}$ ). 7.01 (brd $2 \mathrm{H}, \mathrm{D}_{2} \mathrm{O}$ exchangeable). 5.35 ( $\mathrm{s}, 1 \mathrm{H}, \mathrm{D}_{2} \mathrm{O}$ exchangeable). 5.09 (d. $J=4.2 \mathrm{~Hz}, 1 \mathrm{H}, \mathrm{D}_{2} \mathrm{O}$ exchangeable). 3.99 (dd $J=6.2$ and $2.2 \mathrm{~Hz}, \mathrm{lH}) .3 .71-3.60(\mathrm{~m} .4 \mathrm{H}) .3 .32(\mathrm{~d} . J=6.2 \mathrm{~Hz} . \mathrm{lH})$, $1.69-1.59(\mathrm{~m}, 3 \mathrm{H}) .0 .81(\mathrm{~m}, 18 \mathrm{H}), 0.01(\mathrm{~m}, 12 \mathrm{H}) .{ }^{13} \mathrm{C} \mathrm{NMR}$ (DMSO- $d_{6} .75 \mathrm{MHz}$ ): $\delta$ 165.7. 155.8. 143.4.93.9.85.5.71.0. 66.2. 65.2. 52.1, 32.2, 25.7, 18.6. 16.3. -5.54. Analy'sis for $\mathrm{C}_{33} \mathrm{H}_{45} \mathrm{~N}_{3} \mathrm{O}_{5} \mathrm{Si}_{2}$ (499.79). Calcd: C. 55.27: H. 9.08: N. 8.41: Found: C. 55.40: H, 9.15: N. 8.53. Compound 15: 'H NMR (DMSO- $d_{6} .300 \mathrm{MHz}$ ): $\delta 7.5 \mathrm{l}(\mathrm{d}, J=7.0 \mathrm{~Hz}, 1 \mathrm{H}$ ) .7 .03 (brd. 2H. $\mathrm{D}_{2} \mathrm{O}$ exchangeable). 5.41 (brs. $1 \mathrm{H} \mathrm{D}_{2} \mathrm{O}$ exchangeable). 5.10 (brs. $1 \mathrm{H} . \mathrm{D}_{2} \mathrm{O}$ exchangeable) 4.01 (d. $\left.J=4.8 \mathrm{~Hz}, \mathrm{lH}\right) .3 .69$ (d. $J=10.6 \mathrm{~Hz}, \mathrm{IH}), 3.60-3.4 \mathrm{l}(\mathrm{m} .3 \mathrm{H}) .3 .35(\mathrm{~d} . J=6.6 \mathrm{~Hz}, \mathrm{lH})$. $1.67(\mathrm{~m} . \mathrm{lH}) .1 .59-1.51(\mathrm{~m} .2 \mathrm{H}) .0 .82(\mathrm{~m} . \mathrm{l} \mathrm{H}) .0 .02(\mathrm{~m} . \mathrm{l} 2 \mathrm{H})$. ${ }^{13} \mathrm{C}$ NMR (DMSO- $d_{6} .75 \mathrm{MHz}$ ): ò $165.5,156.1,1+6.3 .92 .7$. 86.1, 71.6, 67.1, 64.9, 53.6, 34.0, 25.1, 18.5, 17.9, -5.58. Analy sis for $\mathrm{C}_{23} \mathrm{H}_{45} \mathrm{~N}_{3} \mathrm{O}_{5} \mathrm{Si}_{2}$ (499.79). Calcd: C. 55.27: $\mathrm{H}$. 9.08: N. 8.41: Found: C. 55.19: H. 8.99: N. 8.34.

ret-(1'R,2'S,3'S,4'R)-9-[2,4-Bis-(tert-butyldimethylsilyloxmethyl)-2,3-dihydroxy-cyclopentan-1-yl]adenine (14) and ret-

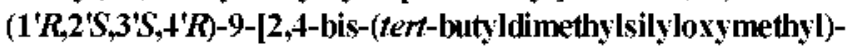
2,3-dihydroxy-cyclopentan-1-yl]adenine (16). The adenine mlcleoside analogues 14 and 16 were syththesized from 13 by the same procedure as described for the preparation of $\mathbf{1 3}$ and 15. Compound 14: y ield $52 \%$. ${ }^{1} \mathrm{H}$ NMR (DMSO- $d_{6 .} 300 \mathrm{MHz}$ ):
8.21 (s, lH). 8.13 (s. 1H). 7.22 (brs. $2 \mathrm{H}$. D $2 \mathrm{O}$ exchangeable), 5.32 (brs. $1 \mathrm{H}, \mathrm{D}_{2} \mathrm{O}$ exchangeable), 5.08 (d, $J=4.2 \mathrm{~Hz}, 1 \mathrm{H} . \mathrm{D}_{2} \mathrm{O}$ exchangeable). $3.96(\mathrm{dd}, J=6.2$ and $4.0 \mathrm{~Hz}, 1 \mathrm{H}$ ). $3.68-3.59$ $(\mathrm{m}, 4 \mathrm{H}), 3.35(\mathrm{~d}, J=7.0 \mathrm{~Hz}, 1 \mathrm{H}), 1.76-1.68(\mathrm{~m} .2 \mathrm{H}) .1 .68(\mathrm{nt}$, lH). $0.81(\mathrm{n}, 18 \mathrm{H}), 0.02(\mathrm{~m}, 12 \mathrm{H}) .{ }^{13} \mathrm{C}$ NMR (DMSO- $d_{6,75}$ $\mathrm{MHz})$ : ô 155.8. 152.5, 149.8, 141.5, 119.7. 87.1. 71.5, 65.3, $64.2,55.2,33.7,25.6 .18 .4 .16 .7 .5 .6$. Analysis for $\mathrm{C}_{24}$ $\mathrm{H}_{45} \mathrm{~N}_{5} \mathrm{O}_{4} \mathrm{Si}_{2}$ (523.82). Calcd.: C. 55.03: H, 8.66: N, 13.37; Found: C. 54.93: H. 8.56: N. 13.44. Compound 16: yield 16\%: ${ }^{\mathrm{l}} \mathrm{H}$ NMR (DMSO-ds. $300 \mathrm{MHz}$ ): ô 8.18 (s. $1 \mathrm{H}$ ), 8.09 (s. lH), 7.15 (brs, 2H. $\mathrm{D}_{2} \mathrm{O}$ exclangeable), 5.38 (brs, 1H. $\mathrm{D}_{2} \mathrm{O}$ exchangeable). 5.11 (d. $J=4.4 \mathrm{~Hz}, 1 \mathrm{H}, \mathrm{D}_{2} \mathrm{O}$ exchangeable). 3.89 (d, $J=6.0 \mathrm{~Hz}, 1 \mathrm{H}), 3.65-3.53(\mathrm{~m} .4 \mathrm{H}), 3.29(\mathrm{~d} . J=6.8 \mathrm{~Hz} .1 \mathrm{H})$, $1.69-1.61(\mathrm{~m} .3 \mathrm{H}), 0.82(\mathrm{~m}, 18 \mathrm{H}), 0.01(\mathrm{~m}, 12 \mathrm{H}) \cdot{ }^{12} \mathrm{C}$ NMR (DMSO- $\left.d_{6}, 75 \mathrm{MHz}\right): \delta 155.6 .153 .0 .148 .9,140.4,118.2,86.7$. $70.8 .66 .0,65.2 .54 .9,32.8,25.7 .18 .7,17.2 .-5.8$. Analysis for $\mathrm{C}_{24} \mathrm{H}_{45} \mathrm{~N}_{5} \mathrm{O}_{4} \mathrm{Si}_{2}(523.82)$. Calcd : C, 55.03; H. 8.66: N. 13.37; Found: C. 55.12: H, 8.73: N, 13.27 .

rel-(1'R,2'S,3'S,t'R)-1-[2,4-Bis-(hydroxymethyl)-2,3-(lihydroxy-cyclopentan-1-yl]cytosine (17). TBAF (0.75 mL, 1.0 M solution in THF. $0.75 \mathrm{mmol}$ ) was added to a solution of $\mathbf{1 3}$ (125 mig, 0.25 nmol) in cosolvent $\left(3.0 \mathrm{~mL}, \mathrm{THF} / \mathrm{CH}_{3} \mathrm{CN} 1: 1\right.$ $v / v)$ at $0{ }^{\circ} \mathrm{C}$. The mixture was stirred overnight at room temperature and then evaporated under reduced pressure. The residue was purified by column chromatography (silica gel. $\left.\mathrm{MeOH} / \mathrm{CH}_{2} \mathrm{Cl}_{2} 1: 4 \mathrm{v} / \mathrm{v}\right)$ to yield $17(55 \mathrm{mg}, 81 \%$ ) as a white solid: $\mathrm{mp} 195 \cdot 197^{\circ} \mathrm{C}$ : UV $\left(\mathrm{H}_{2} \mathrm{O}\right)$ : $\lambda_{\max } 270.5 \mathrm{~nm} .{ }^{l} \mathrm{H}$ NMR (DMSO- $d_{6 .} 300 \mathrm{MHz}$ ): 7.43 (d. $J=6.9 \mathrm{~Hz} . \mathrm{lH}$ ). 7.02 (brd. $2 \mathrm{H}, \mathrm{D}_{2} \mathrm{O}$ exchangeable). 5.59 (d. $\left.J=7.0 \mathrm{~Hz} . \mathrm{HH}\right), 5.36$ (brs. $1 \mathrm{H}$. $\mathrm{D}_{2} \mathrm{O}$ exchangeable), $5 . \mathrm{l}+$ (brs. $\mathrm{IH}, \mathrm{D}_{2} \mathrm{O}$ exchangeable) 4.89 (t, $J=4.2 \mathrm{~Hz}, 1 \mathrm{H}, \mathrm{D}_{2} \mathrm{O}$ exchangeable), 4.80 (brs. $1 \mathrm{H}, \mathrm{D}_{2} \mathrm{O}$ exchangeable). $3.65-3.57(\mathrm{~m}, 3 \mathrm{H}) .3 .40-3.33(\mathrm{~m}, 2 \mathrm{H}), 3.28(\mathrm{~d}$. $J=6.2 \mathrm{~Hz}, \mathrm{lH}), 1.69-1.62(\mathrm{~m} .2 \mathrm{H}) .1 .53$ (dd $J=8.2$ and 6.8 $\mathrm{Hz}, 1 \mathrm{H}$ ) ${ }^{13} \mathrm{C}$ NMR (DMSO- $d_{6}, 75 \mathrm{MHz}$ ): ô 165.6. 155.t, 144.7. $94.4,85.2 .70 .6,63.8,61.2,52.6,32.3 .17 .4$. Analysis for $\mathrm{C}_{11} \mathrm{H}_{1}: \mathrm{N}_{5} \mathrm{O}_{5}\left(+1.5 \mathrm{H}_{2} \mathrm{O}, 317.29\right)$. Calcd.: C. 44.29; H. 6.76: $\mathrm{N}$, 14.08: Found: C. 44.27: H, 6.67: N, 13.96.

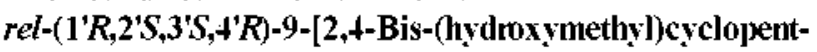
2-en-1-yl] adenine (18). Adenine nucleoside analogue 18 was synthesized from 14 by the same procedure as described for the preparation of 17 as a white solid: yield $75 \% ; \mathrm{mp} 202-205$ ${ }^{\circ} \mathrm{C}: \mathrm{UV}\left(\mathrm{H}_{2} \mathrm{O}\right): \lambda_{\max } 260.0 \mathrm{~nm} .{ }^{1} \mathrm{H}$ NMR (DMSO- $d_{6}, 300 \mathrm{MHz}$ ): $\delta 8.17(\mathrm{~s}, 1 \mathrm{H}), 8.05(\mathrm{~s}, 1 \mathrm{H}), 7.12$ (brd. $2 \mathrm{H}$. $\mathrm{D}_{2} \mathrm{O}$ exchangeable). 5.29 (brs. IH, D $2 \mathrm{O}$ exchangeable). 5.12 (d. $J=4.6 \mathrm{~Hz}, \mathrm{IH}_{3} \mathrm{D}_{2} \mathrm{O}$ exchangeable). $4.86\left(\mathrm{t} . J=4.2 \mathrm{~Hz}, \mathrm{IH} . \mathrm{D}{ }_{2} \mathrm{O}\right.$ exchangeable) 4.79 (t, $J=4.0 \mathrm{~Hz}, 1 \mathrm{H} . \mathrm{D}_{2} \mathrm{O}$ exchangeable), 3.81 (dd, $J=10.8$ and $6.8 \mathrm{~Hz} .2 \mathrm{H}), 3.68-3.53(\mathrm{~m}, 3 \mathrm{H}) .3 .3 \mathrm{l}(\mathrm{d} . J=6.2 \mathrm{~Hz}, \mathrm{lH}) .1 .89-$ 1.80 (n. 2H). 1.69 (m. lH). ${ }^{13} \mathrm{C}$ NMR (DMSO-d. $75 \mathrm{MHz}$ ): ô $155.7,152.6 .146 .5 .143 .2,117.9 .84 .9,71.3,63.6,61.3 .52 .9$. 31.8, 16.9. Analysis for $\mathrm{C}_{12} \mathrm{H}_{1}: \mathrm{N}_{5} \mathrm{O}_{4}(+0.5 \mathrm{MeOH}, 311.31)$ : $\mathrm{C}$, 48.23: H. 6.15: N. 22.49; Found: C, 48.17: H. 6.09: N. 22.53

\section{References}

1. Gordon, C. P.; Keller, P. A. J. Wed Chem 2005, 48,1 .

2. De Francesco, R: Migliaccio, G. Natme 2005, 436,953 .

3. De Clercq. E. Nat. Rev. Ding Discov. 2007, 6, 1001

4. Bera, S.; Malik, L.; Bhat, B.; Carroll, S. S.; MacCoss, M.; Olsen, 
D. B.: Tomassini, J. E.; Eldrup, A. B. Bioong. Med. Chem. Lett. $2003,13,4455$.

5. Eldrup, A. B.: Prhave, M.: Brooks, J.: Bhat, B.: Prakash, T. P.: Song, Q; Bera, S.; Bhat, N.; Dande, P; Cook, P. D.; Bemett, C. F.: Carroll, S. S.; Ball, R. G.; Bosserman, M.; Burlein, C.; Colwell, L. F.; Fay, T. F.; Flores, O A.: Getty, K.; LaFemina, R. L.: Leone, I.: MacCoss, M.; McMasters, D. R.: Tomassini, J. E.: Von Lan1gen, D: Wolanski, B.; Olsen, D. B. J. Hed. Chent. 2004, $47,5284$.

6. Clark, J. L.; Hollecker, L.; Mason, J. C.; Stuyver, L. . T.; Thamish, P. M.; Lostia, S.; McBrayer, T. R.; Schinazi, R. F.: Watanabe, K. A.; Otto, M. I.; Furnan1, P. A.; Stec, W. J.: Patterson, S. E.; Pan1kiewicz, K. W. J. Afed Chem 2005, $48,5504$.

7. Arimilli, M. N.; Doughertv, I. P; Cundv, K. C.; Bischofberger, N. In Advances in thtiviral Dng Desigm. De Clerca. E., Ed. Jai Press Inc: Stanford Connecticut, 1999; vol. 3, pp 69-91.

8. El Kouni, M. H. Curr Pham. Design 2002, 8,581

9. Eldrup, A. B.; Allerson, C. R.; Bennett, C. F.; Bera, S.; Bhat, B.; Bhat, N.; Bosserman, M. R.; Brooks, T.: Burlein, C. Carrol, S. S. Cook, P. D.: Getty, K. L.; MacCross, M.: McMasters, D. R.: Olsen, D. B.; Prakash, T. P.: Prhave, M.: Song. Q. L.; Tomassini, J. E.; Xia, J. J. Afed. Chem. 2004, 47, 2283.
10. Canol, S. S.: Tomassini. I. E.: Bosseman, M.: Getty, K.; Stahllhut, M. W.: Eldrup, A. B.: Bhat, B.: Hall, D.; Simcoe, A. L:: LaFemina, R.; Rutkowski, C. A.: Wolanski, B.; Yang, Z.: Migliaccio, G.; De Francesco, R.; Kuo, L. C.; MacCross, M.; Olsen, D. B. J. Biol. Chem. 2003, 278, 11979.

11. Yoo, B. N.: Kim, H. O:: Moon, H. R.: Seol, S. K.: Tang, S. K.: Lee, K. M.; Teong, L. S. Bioong. Med. Chem. Lett. 2006, 16, 4190.

12. Kim, J. W; Hong, J. H. Arch. Pham. Chem. Life Sci. 2005, 338 , 399.

13. Nakata, M.; Enari, H.; Kinoshita, M. Bull. Chem. Soc. Jpm. 1982, 55,3283 .

14. Furstner, A. Angew. Chem. 2000, 39, 3012.

15. Prunet, J . Angew. Chem. 2003, $12,2826$.

16. Rivkin, A.: Cho, Y. S.: Gabarda, A. E.: Yoshimura, F.: Danishefshy, S. T. J. Nat. Prod 2004, 67, 139.

17. Deiter, A.; Martin, S. F. Chent Rev. 2004, 104, 2199.

18. Gaich, T.; Mulzer, J. Curr Top. Hed. Chem 2005, $5,1473$.

19. Brown, B.; Hegedus, L. S. J. Org. Chem. 2000, 65, 1865.

20. Burlina, F; Favre, A.; Fourrey, T.-L. Bioorg. Hed. Chent. Lent. 1997, 7, 247. 